\title{
Evaluación de pavimentos en base a métodos no destructivos y análisis inverso
}

\author{
Edisson Avila, Flavio Albarracín, Jaime Bojorque \\ Facultad de Ingeniería, Universidad de Cuenca, Av. 12 de abril s/n, Cuenca, Ecuador. \\ Autores para correspondencia: edisonavil45@ hotmail.es, flavioalbarracin@ hotmail.com \\ Fecha de recepción: 12 de enero de 2015 - Fecha de aceptación: 8 de marzo de 2015
}

\section{RESUMEN}

En este documento se analiza la factibilidad y eficiencia de la deflectometría de impacto para evaluar la capacidad portante de la estructura de un pavimento flexible en vías locales de la red austral E40. Mediante el equipo no destructivo conocido como deflectómetro de impacto se generan deflexiones en el pavimento, las que son interpretadas a través de técnicas basadas en análisis inverso como AASHTO 93, LUKANEN, YONAPAVE y ROHDE. Dichas técnicas permiten la cuantificación y cualificación del nivel de deterioro estructural del sistema pavimento-subrasante a través de dos parámetros. El primer parámetro se define mediante el módulo resiliente, el cual es comparado con los softwares EVERCALC y ANSYS, que sustentan su desarrollo en análisis inverso y elementos finitos respectivamente. Por otro lado, el segundo parámetro se define mediante la capacidad estructural del sistema pavimento-subrasante, el cual es analizado a través del concepto de deflexión característica propuesto por la guía de diseño AASHTO 93. EL estudio da a conocer que el mejor método para caracterizar el estado del pavimento es la técnica propuesta por YONAPAVE. El documento no solo presenta resultados de la condición actual de la vía bajo estudio sino también proporciona recomendaciones con respecto al manejo del pavimento de la vía, que permitan transformar la ingeniería de pavimentos a una ingeniería de gestión de los pavimentos.

Palabras clave: Técnica de auscultación, deflexión, análisis inverso, ANSYS, EVERCALC, elementos finitos.

\begin{abstract}
The feasibility and efficiency of impact deflectometry, to assess the structural capacity of flexible pavements on local roads of the southern E40 road network, were analyzed. Deflections generated by the impact of the non-destructive Falling Weight Deflectometer are interpreted using back-calculation techniques, such as proposed by AASHTO 93, LUKANEN, YONAPAVE and ROHDE. Those techniques enable the quantification and qualification of the structural deterioration level of existing pavement-subgrade systems by two parameters. The first parameter is derived from the resilient modulus, and its value is compared to the output of inverse and finite element analysis using respectively the EVERCALC and ANSYS software. The second parameter is defined by the structural capacity of the pavement-subgrade system, and derived applying the concept of characteristic deflection as described in the AASHTO Design Guide 93. The study revealed that the best method to characterize the road status is the analysis technique proposed by YONAPAVE. The approach not only presents results of the current condition of the road under study, but provides also recommendations regarding the management of road pavement. The developed methodology enables to elevate in future pavement engineering to pavement management engineering.
\end{abstract}

Keywords: Auscultation techniques, deflection, back-calculation, ANSYS, EVERCALC, finite element. 


\section{INTRODUCCIÓN}

Debido a que las redes viales de un país son las que promueven un verdadero desarrollo social, cultural y económico de sus habitantes, el estudio de la condición actual de los pavimentos ha sido y continuará siendo un mecanismo importante para mantener un adecuado nivel de servicio; el cual relaciona la integridad estructural de un pavimento con la comodidad de manejo de los usuarios viales (Montejo Fonseca, 2002).

Generalmente, la medición del estado de un pavimento existente requería de la aplicación de procesos destructivos en la vía, lo que alteraba el equilibrio del sistema pavimento-subrasante. Sin embargo, a través de nuevas tecnologías o también llamados instrumentos de última generación que aplican técnicas para ensayos de alto rendimiento, conocidas como pruebas no destructivas (NonDestructive Test, NDT), se ha mejorado la auscultación de las vías. De entre estas técnicas se destaca la utilización del deflectómetro de impacto (Falling Weight Deflectometer, FWD), cuya función es la de analizar la condición estructural de los pavimentos mediante la interpretación de las deflexiones producidas bajo cargas dinámicas que simulan el efecto del tránsito. Por este motivo, agencias dedicadas a la investigación, como la American Association of State Highway and Transportation Officials (AASHTO) y Washington State Department of Transportation (WSDOT), han establecido modelos matemáticos para correlacionar dichas tecnologías a través de parámetros o indicadores de estado del pavimento. Estos nuevos métodos proporcionar soluciones de uso directo que permitan establecer un adecuado mantenimiento, rehabilitación o reconstrucción de la estructura.

En el presente estudio, se utilizan como principales guías o documentos, la guía de diseño de la AASHTO (1993), del Instituto del Asfalto (2000), de la ASTM-D4695-03 (2003) y la D4694-96 (1996) para conocer el proceso de evaluación estructural aplicando deflectometría de impacto. A través de cada una, se analiza la factibilidad y eficiencia de la técnica de la deflectometría de impacto para evaluar la capacidad portante de la estructura del pavimento en vías locales; todo ello con la realización de ensayos de deflexión generados por el impacto provocado por este equipo en el pavimento. La información dada por el equipo, se complementa con pruebas destructivas como son la extracción de núcleos y la realización de calicatas para conocer los espesores de la capa de rodadura como de las capas granulares. Entonces se realiza una comparación entre los modelos matemáticos propuestos por AASHTO 93 (1993), LUKANEN (Lukanen \& Han, 1992), YONAPAVE (Hoffman, 2003) y ROHDE (Rohde, 1994), estableciendo las ventajas y desventajas de cada método. Dicha comparación se realiza mediante el cálculo del módulo resiliente de subrasante y de la capacidad estructural del pavimento a través del número estructural $(\mathrm{SN})$. Con la implementación del programa computacional EVERCALC que trabaja con un proceso de análisis inverso desarrollado por WSDOT (2005) y de ANSYS (Roa Garzón \& Garzón Alvarado, 2012), que emplea la teoría de los elementos finitos, se contrarrestan los resultados obtenidos por los 4 modelos matemáticos mencionados. Se plantea determinar que el modelo propuesto por YONAPAVE basado en análisis inverso es una metodología que brinda una adecuada caracterización del pavimento-subrasante en base a las características de los materiales. Finalmente, habiendo establecido el modelo matemático más adecuado se determina la condición estructural actual de la vía mediante datos del tráfico proporcionados por el Ministerio de Transporte y Obras Públicas (MTOP).

La síntesis del estudio se expone a continuación:

- A través de las Tablas 1, 2 y 3 se establecen los parámetros bajo los que se definen cada uno de los modelos matemáticos AASHTO 93, LUKANEN, YONAPAVE y ROHDE.

- En la Figura 2 se presenta el equipo de deflectometría de impacto FWD, mediante el cual se registran las deflexiones obtenidas a lo largo de la vía. Dicho registro es seccionado a través de tramos homogéneos, en donde se toma como referencia la deflexión máxima, representándose este procedimiento con la Figura 5.

- Una vez realizado el seccionamiento de la vía, el cuenco de deflexiones es ingresado al software EVERCALC para caracterizar la estructura del pavimento mediante el módulo elástico de cada capa, y por ende encontrar un módulo resiliente de subrasante para cada tramo de análisis, el mismo que se presenta en la Figura 6. Además para realizar una verificación de los resultados se usa el software ANSYS que sustenta su desarrollo en el método de elementos 
finitos (Figs. 3 y 4) por lo que se comparan las curvas de deflexión halladas tanto por ANSYS como las medidas por el FWD en puntos característicos correspondientes a cada sección como se muestra en la Figura 7.

- En la Figura 8 se efectúa una comparación del módulo resiliente de subrasante evaluado por los 4 modelos, y en la Figura 9 en cambio se compara el número estructural efectivo $\left(\mathrm{SN}_{\mathrm{ef}}\right)$.

- Habiéndose encontrado el módulo resiliente de la subrasante con cada uno de los modelos matemáticos planteados, se los compara con el obtenido a través de EVERCALC, cuyos resultados son expuestos en la Tabla 5.

- De la misma forma, la capacidad estructural $\mathrm{SN}_{\text {ef }}$ de los modelos, ha sido comparada con la capacidad estructural efectiva que se encuentra en función de la deflexión característica, datos que se presentan en la Tabla 6 .

- Mediante los dos últimos ítems mencionados se establece que el modelo de YONAPAVE es una metodología adecuada para la evaluación estructural de los pavimentos, y por ello finalmente se calcula la capacidad estructural que se encuentra en función del tráfico presente en la vía de estudio, resultados que se muestran en la Tabla 7.

\section{MATERIALES}

La auscultación se entiende como un procedimiento esencial para determinar el estado actual de la vía tanto funcional como estructural, utilizando para ello equipos y herramientas tecnológicas. En una vía, la aplicación de carga se debe principalmente al tránsito vehicular de donde la respuesta del pavimento ante la aplicación de dicha carga, produce un estado de tensiones y deformaciones, con lo cual los desplazamientos que ocurren son en sentido vertical en magnitudes muy pequeñas del orden de centésimas o milésimas de milímetro (AASHTO, 1993). Dicho desplazamiento es conocido como deflexión. Es necesario entender que ante la aplicación de una carga vehicular, si bien se produce una deflexión máxima sobre el punto de aplicación, alrededor de esta zona también se produce desplazamientos verticales de la superficie al que se lo conoce como cuenco de deflexión.

Con la finalidad de conocer el estado actual de la vía se emplean dos conceptos fundamentales. El primero es una técnica de evaluación basada en análisis inverso, la cual partiendo del resultado u objetivo al que se quiere llegar, procede a analizar cuáles son los factores o causas de los que depende dicho resultado, y de este modo mediante un proceso iterativo caracterizar el problema, sin tener que utilizar medios invasivos para este estudio. El análisis inverso es una evaluación mecanicista de la superficie del pavimento y del cuenco de deflexiones generada por diversos aparatos de carga dinámica o estática, involucra el cálculo de deflexiones teóricas asumiendo una primera aproximación para los módulos de cada capa, luego mediante un proceso iterativo se ajustan las curvas de deformación teóricas y las curvas medidas por el FWD, hasta que se presente un ajuste razonable (Corros et al., 2009). Los módulos hallados de esta manera se consideran representativos de la respuesta del pavimento frente a la carga.

El segundo concepto se define como la modelación, siendo este un proceso de abstracción del sistema real a un modelo cuantitativo, en el que se representa la estructura de un pavimento como un conjunto de capas de espesor constante y longitud infinita horizontal, las que se encuentran apoyadas sobre un dominio semi-infinito o subrasante (Rodriguez Calderón \& Pallarez Muñoz, 2009). Por lo que, un modelo matemático es una construcción matemática abstracta y simplificada relacionada con una parte de la realidad.

\subsection{Zona de estudio}

La investigación se llevó a cabo en la Vía Chicti-Sevilla de Oro que cuenta con una longitud de $27 \mathrm{~km}$ y un ancho de $7 \mathrm{~m}$, la cual es parte constituyente de la carretera El Descanso - Paute - Guarumales; correspondiente a la Transversal Austral E40 que recorre la Zona Oriental de la Provincia del Azuay. Dicha carretera entró a un plan de estudios de rehabilitación y reconstrucción, por lo que en agosto del 
2010 inició con la operación; desde esa fecha hasta la actualidad, la vía existente se ha encontrado en continua operación. La estructura de pavimento existente en la vía está compuesta por una capa de rodadura de concreto asfáltico, la que se asienta sobre una base granular, y esta a su vez se asienta sobre una capa de subbase; permitiendo que la estructura sea soportada por la subrasante o terreno natural. En la Figura 1 se observa la vía en estudio (línea roja), en donde se llevaron a cabo las respectivas pruebas de deflectometría para determinar la condición estructural.

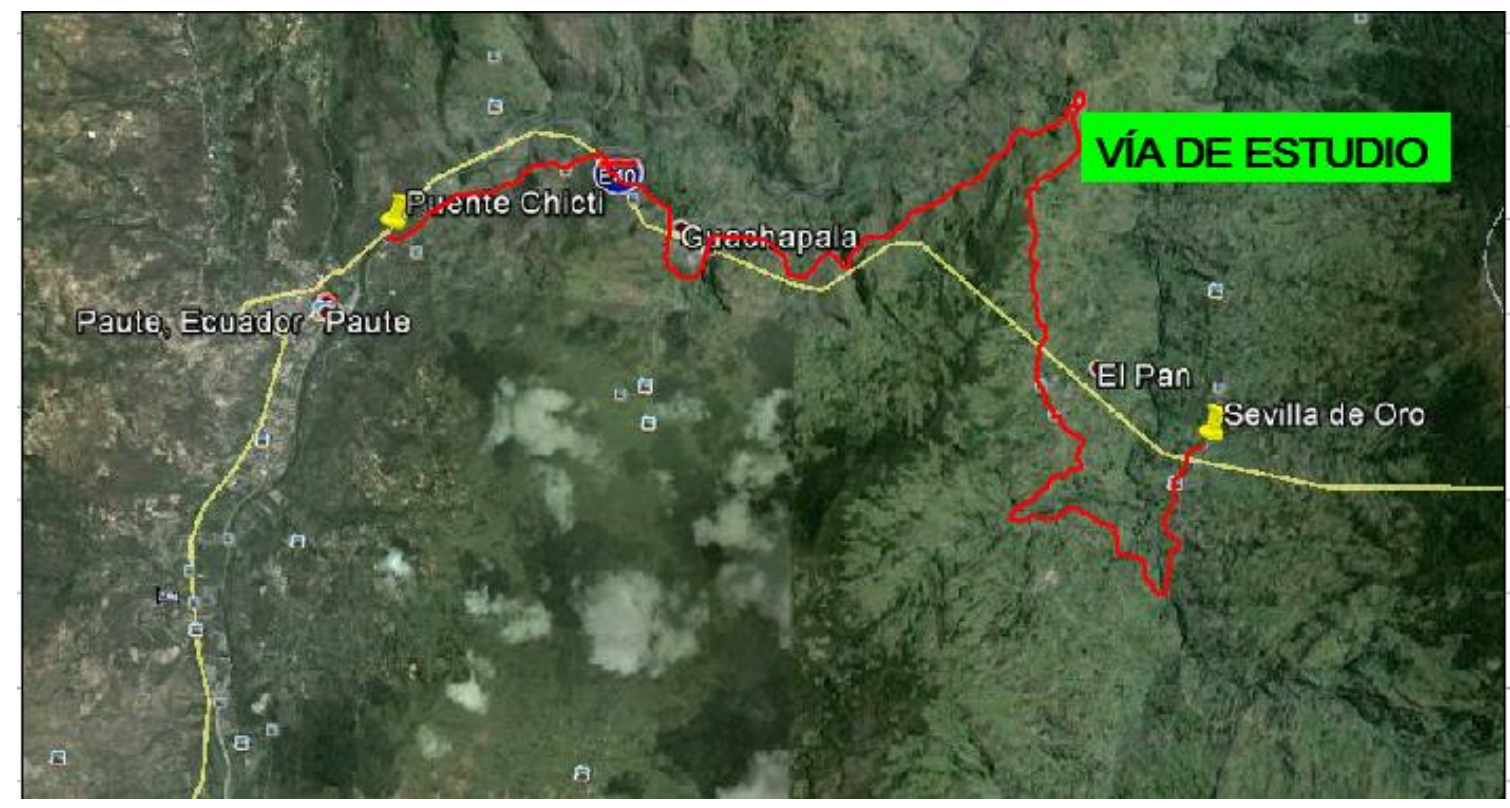

Figura 1. Localización del tramo vial CHICTI-SEVILLA DE ORO.

\section{MÉTODOS}

\subsection{Deflectometría de Impacto}

El FWD es un equipo utilizado en la Ingeniería de Pavimentos para evaluar la condición estructural del pavimento. El equipo de ensayo puede ser montado en un vehículo o en un sistema de remolque tirado por un vehículo. La adquisición de un equipo sofisticado como éste, puede resultar costosa pero presenta un alto rendimiento, es automatizado y funciona bajo una carga dinámica (Murillo Feo \& Bejarano Urrego, 2013). Por carga dinámica se entiende una mejor simulación de las cargas del tráfico sobre el pavimento. El equipo cuenta con una serie de sensores o geófonos (10 geófonos) colocados en una regleta, los que se encuentran predispuestos a diferentes distancias medidas desde el centro del plato de carga, el cual tiene un diámetro de $30 \mathrm{~cm}$. La función de dichos sensores es medir las deflexiones producidas al aplicar la carga dinámica sobre un punto determinado y de este modo generar un cuenco de deflexión (véase Fig. 2), a través del cual se puede caracterizar la capacidad estructural del sistema subrasante-pavimento.

\subsection{Modelos matemáticos utilizados para la caracterización de la subrasante}

La auscultación estructural de pavimentos, empleando deflectometría de impacto, tiene como finalidad determinar la capacidad portante del sistema pavimento-subrasante en cualquier momento de su vida útil; así como el de establecer y cuantificar la necesidad de realizar o no rehabilitaciones (Guzmán Suárez, 2009), mediante modelos matemáticos propuestos por diferentes entidades tales como la AASHTO (1993), LUKANEN (Lukanen \& Han, 1991), YONAPAVE (Hoffman, 2003), WSDOT (2005) ó RODHE (Rodhe, 1994). Mediante estos modelos es posible hallar parámetros como el número estructural efectivo $\left(\mathrm{SN}_{\mathrm{ef}}\right)$ y el módulo resiliente de la subrasante $\left(\mathrm{M}_{\mathrm{r}}\right)$; los cuales son necesarios para el diseño y rehabilitación de las carreteras. A continuación se presenta la 
conceptualización básica de los modelos matemáticos, los cuales son la base fundamental para la evaluación estructural del pavimento.

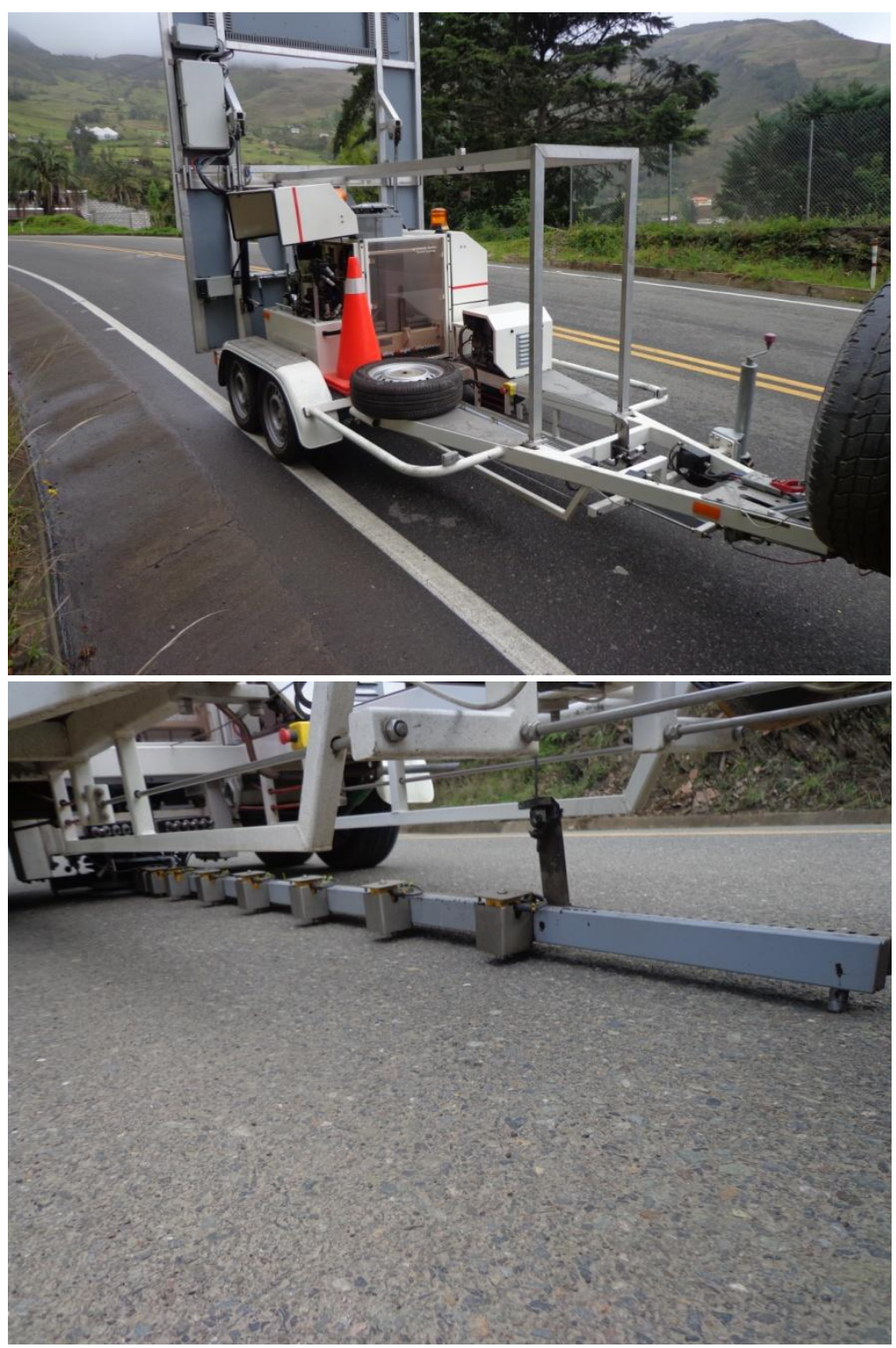

Deflectómetro de impacto

(FWD).

Disposición de los geófonos en el FWD.

Figura 2. Deflectómetro de Impacto - PRIMAX FWD del Ministerio de Transporte y Obras Públicas.

\section{Método de AASHTO 93}

La Ec. 1 permite el cálculo del módulo resiliente de la subrasante por retro-cálculo $\left(\mathrm{M}_{\mathrm{rr}}\right)$ conociendo la magnitud de carga y una deflexión medida a cierta distancia del centro del plato de carga.

$$
M_{r r}=\frac{0.24 P}{d_{r} * r}
$$

dónde: $\mathrm{M}_{\mathrm{rr}}$ es el módulo resiliente de la subrasante obtenido por retro-cálculo (psi); $\mathrm{P}$ es la carga aplicada (lb); $\mathrm{d}_{\mathrm{r}}$ es la deflexión a la distancia $\mathrm{r}$ medida desde el centro del plato de carga (in) y $\mathrm{r}$ es la distancia desde el centro del plato de carga (in).

Para la determinación del módulo $\mathrm{M}_{\mathrm{rr}}$ se utiliza una deflexión medida a cierta distancia tal, la cual debe estar lo suficientemente lejos para que proporcione una buena estimación del valor de dicho parámetro, pero también lo suficientemente cerca para evitar una deflexión muy pequeña la cual no 
sería significativa (AASHTO, 1993). Según la metodología de AASHTO la mínima distancia a la que se debe considerar la deflexión, se expresa como:

$$
a_{e}=\sqrt{\left[a^{2}+\left(H_{T} \sqrt[3]{\frac{E_{P}}{M_{r r}}}\right)^{2}\right]}
$$

dónde: $\mathrm{H}_{\mathrm{T}}$ es el espesor de la estructura del pavimento (in); $\mathrm{E}_{\mathrm{p}}$ es el módulo elástico de la estructura del pavimento (psi); a es el radio del plato de carga (in); y $\mathrm{a}_{\mathrm{e}}$ es el radio del bulbo de tensión, en la interface subrasante-pavimento (in).

Los valores de $\mathrm{M}_{\mathrm{rr}}$ son ajustados para ser consistentes con los obtenidos por ensayos de laboratorio. AASHTO 93 recomienda que los valores de $\mathrm{M}_{\mathrm{rr}}$ deben ser multiplicados por un factor de ajuste (C) igual a 0.33 debido a que dichos valores son superiores a los obtenidos en laboratorio (AASHTO, 1993), obteniendo el $\mathrm{M}_{\mathrm{r}}$.

$$
M_{r}=C\left(\frac{0.24 P}{d_{r} * r}\right)
$$

Una vez conocido el Módulo $\mathrm{M}_{\mathrm{rr}}$, y el espesor total de la estructura del pavimento $\left(\mathrm{H}_{\mathrm{T}}\right)$, la cual se asienta sobre la subrasante; se puede calcular el Módulo Elástico del pavimento $\left(\mathrm{E}_{\mathrm{p}}\right)$, dicho parámetro está determinado por el valor de la deflexión medida en el centro del plato de carga $\left(\mathrm{D}_{\mathrm{o}}\right)$ mediante la Ec 5:

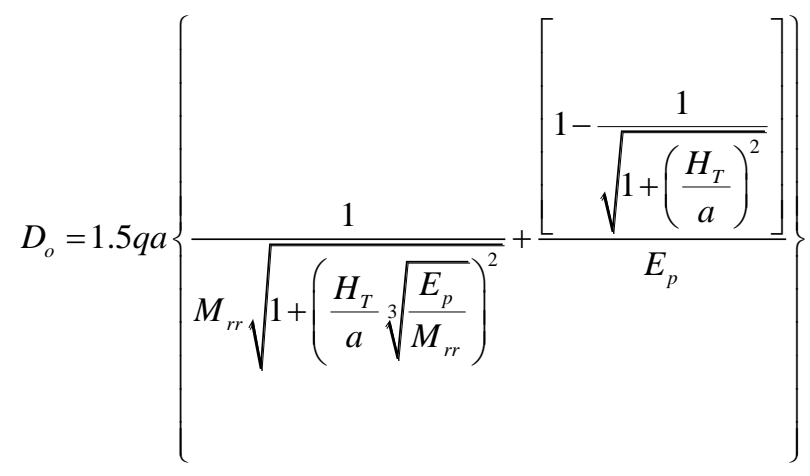

La variable más importante en la metodología AASHTO para el diseño de pavimentos es el número estructural efectivo $\left(\mathrm{SN}_{\mathrm{ef}}\right)$, este valor junto con la proyección de tránsito en el periodo de diseño permite determinar espesores de la carpeta de rodadura o análisis de vida residual del pavimento.

$$
S N_{e f}=0.0045 H_{T} \sqrt[3]{E_{p}}
$$

donde: $\mathrm{D}_{\mathrm{o}}$ es la deflexión medida en el centro del plato de carga y ajustada a una temperatura de $20^{\circ} \mathrm{C}$, en pulgadas; $\mathrm{SN}_{\mathrm{ef}}$ es el Número Estructural Efectivo del Pavimento.

\section{Método de LUKANEN}

La evaluación estructural mediante el método de LUKANEN, utiliza las variaciones del modelo de Hogg, para la evaluación del módulo de la subrasante, mediante relaciones obtenidas en investigaciones llevadas a cabo por Minesota Department of Transportation (Mn/DOT; Higuera Sandoval, 2010). El modelo de Hogg está basado en un sistema hipotético de dos capas que consiste en una placa relativamente delgada que se apoya sobre una fundación elástica (del Aguila Rodriguez, 2007). El modelo define dos parámetros, la distancia donde la deflexión es la mitad de la deflexión central $\left(\mathrm{r}_{50}\right)$, y la longitud característica $\left(\mathrm{l}_{\mathrm{o}}\right)$ que es la distancia radial en donde se ubica el punto de 
inflexión del cuenco de deflexiones. Dichos parámetros se determinan a partir de las Ecs. (7) y (8) respectivamente.

$$
\begin{gathered}
r_{50}=\frac{A^{C}-B}{\left[A *\left\{\frac{D_{o}}{d_{r}}-1\right\}\right]^{C}-B} \\
l o=\frac{Y r_{50}+\sqrt{\left(Y r_{50}\right)^{2}-4 a X r_{50}}}{2}
\end{gathered}
$$

donde: $\mathbf{r}_{\mathbf{5 0}}$ es la distancia donde la deflexión es la mitad de la deflexión central $(\mathrm{cm}), \mathbf{l}_{\mathbf{0}}$ es la longitud característica (cm); A,B,C,X,Y son los coeficientes de correlación.

Para la determinación de $\mathrm{M}_{\mathrm{rr}}$, se calcula inicialmente la relación entre la rigidez por carga puntual y la rigidez por carga distribuida sobre la superficie del pavimento.

$$
\frac{S_{o}}{S}=1-M *\left(\frac{a}{l o}-0.20\right)
$$

Según el modelo de LUKANEN, $\mathrm{M}_{\mathrm{rr}}$ e se define con:

$$
M_{r r}=\left[\frac{\mathrm{k}^{*} l * P}{l o^{*} \mathrm{Do}}\right] * \frac{S o}{S}
$$

dónde: $\mathrm{S}_{\mathrm{o}} / \mathrm{S}$ es la relación de rigideces por carga puntual y distribuida; $\mathrm{M}$ es el coeficiente para relación de rigideces; 1 es el factor de influencia; $\mathrm{k}$ es el coeficiente numérico que depende del coeficiente de Poisson.

Para determinar el número estructural efectivo se utiliza la Ec. 11, la cual es función de los parámetros determinados a partir del análisis inverso:

$$
S N_{e f}=0.0182 * l_{0} \sqrt[3]{M_{r r}}
$$

En la Tabla 1 se dan a conocer los parámetros que usa el modelo matemático de LUKANEN

Tabla 1. Coeficientes para el método de LUKANEN.

\begin{tabular}{ccccccccccc}
\hline \multicolumn{10}{c}{ Profundidad del estrato rígido (ho/lo) $=10$} \\
\hline Caso & $\mu$ & Dr/Do & $\mathrm{A}$ & $\mathrm{B}$ & $\mathrm{C}$ & $\mathrm{X}$ & $\mathrm{Y}$ & $\mathrm{M}$ & $\mathrm{I}$ & $\mathrm{k}$ \\
\hline I & 0.5 & $>0.7$ & 2.46 & 0 & 0.5920 & 0.183 & 0.620 & 0.52 & 0.1614 & 1.500 \\
& & $<0.7$ & 371.1 & 2 & 0.2190 & 0.183 & 0.620 & 0.52 & 0.1614 & 1.500 \\
II & 0.4 & $>0.426$ & 2.629 & 0 & 0.5480 & 0.192 & 0.602 & 0.48 & 0.1684 & 1.633 \\
& & $<0.426$ & 2283.4 & 3 & 0.2004 & 0.192 & 0.602 & 0.48 & 0.1684 & 1.633 \\
\hline
\end{tabular}

\section{Método de YONAPAVE}

Este método estima el módulo resiliente $\mathrm{M}_{\mathrm{rr}}$ a partir de los valores de deflexión, basado en la teoría del modelo elástico de Hogg (del Aguila Rodriguez, 2007); es decir esta metodología se basa en la interpretación de la forma del cuenco de deflexiones, y toma en cuenta la capacidad portante del pavimento.

Para la determinación del parámetro AREA (área del cuenco de deflexiones en in) se utiliza la siguiente Ec.12:

$$
A R E A=6\left[1+2 \frac{D_{30}}{D_{0}}+2 \frac{D_{60}}{D_{0}}+\frac{D_{90}}{D_{0}}\right]
$$


donde: $\mathrm{D}_{0}, \mathrm{D}_{30}, \mathrm{D}_{60}$ y $\mathrm{D}_{90}$ son las deflexiones obtenidas mediante la aplicación del FWD $(\mu \mathrm{m})$, a distancias de 0, 30, 60 y $90 \mathrm{~cm}$ respectivamente medidas respecto al centro del plato de carga.

YONAPAVE (Guzmán Suárez, 2009) expresa la relación entre la longitud característica (lo) y el área del cuenco de deflexión (AREA) usando la Ec. 13:

$$
l o=A e^{\mathrm{B} A R E A}
$$

dónde: A, B son los coeficientes de ajuste de curva (ver Tabla 2).

Una vez que el valor de la longitud característica es determinado, se procede a calcular $\mathrm{M}_{\mathrm{rr}}$. Este parámetro considera la presión de contacto con la superficie del pavimento, la deflexión que se produce directamente bajo el plato de carga del deflectómetro de impacto y los factores de ajuste de curva que se encuentran en la Tabla 2.

$$
\mathrm{M}_{r r}=\mathrm{m} * \frac{\mathrm{P}}{\mathrm{D}_{0}} * 1_{0}^{\mathrm{n}}
$$

En base a estos parámetros, es posible realizar el cálculo del número estructural efectivo de la subrasante mediante:

$$
S N_{e f}=0.0182 * l_{o} \sqrt[3]{M_{r r}}
$$

Según Guzmán Suárez (2009), Hoffman en 1977 introduce una corrección al $\mathrm{SN}_{\mathrm{ef}}$ a partir de correlaciones encontradas a través de herramientas computacionales de análisis inverso, como es el caso del programa computacional MODULUS (Liu \& Scullion, 2001), con la Ec. 16 de la forma:

$$
S N_{e f}=2\left[0.0182 * l_{o} \sqrt[3]{M_{r r}}\right]-0.5
$$

En la Tabla 2 se presentan los parámetros empleados en el modelo propuesto por YONAPAVE.

Tabla 2. Método de YONAPAVE: Coeficientes de ajuste de curva para determinar la longitud característica (lo).

\begin{tabular}{cccccc}
\hline Rango de valores de área (pulgadas) & $\mathrm{h} / \mathrm{lo}$ & $\mathrm{A}$ & $\mathrm{B}$ & $\mathrm{m}$ & $\mathrm{n}$ \\
\hline Mayor o igual a 23 & 5 & 3.275 & 0.1039 & 926.9 & -0.8595 \\
Mayor o igual a 21 y menor que 23 & 10 & 3.691 & 0.0948 & 1152.1 & -0.8782 \\
Mayor o igual a 19 y menor que 21 & 20 & 2.800 & 0.1044 & 1277.6 & -0.8867 \\
Menor que 19 & 40 & 2.371 & 0.1096 & 1344.2 & -0.8945 \\
\hline
\end{tabular}

\section{Método de ROHDE}

El concepto que maneja este método es que el valor máximo de deflexión $\left(\mathrm{D}_{\mathrm{o}}\right)$ medido a partir del FWD es una combinación de la deflexión en la subrasante y la compresión elástica de la estructura del pavimento (Rohde, 1994). Está autor valora al módulo resiliente de la subrasante a partir de la deflexión del sensor ubicado a $1.8 \mathrm{~m}$ del centro de plato de carga. La expresión que relaciona el módulo de la subrasante con los datos de deflexión se define mediante la Ec.17:

$$
D_{180}=97480\left(1000 M_{r r}\right)^{-1.055}
$$

De esta manera se plantea una relación entre el valor de la deflexión producido a 1.5 veces el espesor total de la estructura del pavimento $\left(\mathrm{H}_{\mathrm{T}}\right)$ con el valor de la deflexión central $\left(\mathrm{D}_{\mathrm{o}}\right)$, la cual se define como el índice estructural del pavimento (SIP: Structural Index of Pavement). Dicho parámetro representa la magnitud de la deformación que ocurre dentro de la estructura de pavimento, la cual se define con la Ec. 18:

$$
S I P=D_{0}-D_{1.5 * H_{T}}
$$


Definiendose el número estructural efectivo como:

$$
S N_{e f}=R_{1} * S I P^{\mathrm{R}_{2}} * H_{T}{ }^{R_{3}}
$$

dónde: $\mathrm{M}_{\mathrm{rr}}$ en $\mathrm{kg} \mathrm{cm}^{-2} ; \mathrm{D}_{1.5 * \mathrm{HT}}$ es la deflexión medida en la superficie del pavimento bajo una carga de $40 \mathrm{KN}$ a una distancia de 1.5 veces el espesor total $\left(\mathrm{H}_{\mathrm{T}}\right)$ de la estructura del pavimento.

En la Tabla 3 se presentan los parámetros empleados en el modelo propuesto por Rohde (1994).

Tabla 3. Coeficientes del método de Rohde para diferentes tipos de superficie.

\begin{tabular}{ccccc}
\hline Tipo de superficie & $\mathrm{R}_{1}$ & $\mathrm{R}_{2}$ & $\mathrm{R}_{3}$ & $\mathrm{r}^{2}$ \\
\hline Sello de asfalto & 0.1165 & -0.3248 & 0.8241 & 0.984 \\
Concreto asfáltico & 0.4728 & -0.4810 & 0.7581 & 0.957 \\
\hline
\end{tabular}

\subsection{Técnicas de análisis inverso y elementos finitos}

La modelación de la estructura del pavimento se realiza mediante dos herramientas computacionales, la primera es EVERCALC basado en análisis inverso desarrollado por el Departamento de Transporte del Estado de Washington (WSDOT, 2005) y la segunda es ANSYS basado en un método racionalista que emplea elementos finitos (Rao Garzón \& Garzón Alvarado, 2012).

\section{Análisis inverso con el programa EVERCALC}

EVERCALC es un programa que estima el módulo elástico de las capas de pavimento, determina esfuerzos y deformaciones a distintas profundidades dentro de la estructura del pavimento (al fondo de la capa asfáltica, a la mitad de las capas granulares y en la parte superior de la subrasante); usando para ello un enfoque netamente iterativo que obtiene los módulos elásticos a partir de una comparación entre las curvas de deflexión calculadas por el programa y las medidas in situ.

Se inicia con una primera estimación de valores de módulo de cada una de las capas, valores que por criterio del usuario son ingresados hasta que el programa converja a una tolerancia permisible o aceptable, la cual se relaciona con los siguientes parámetros: media cuadrática, tolerancia del módulo y un máximo número de iteraciones (WSDOT, 2005), según las Ecs. 20 y 21:

- Media cuadrática (RMS):

$$
R M S(\%)=\sqrt{\frac{1}{n_{d}} \sum_{i=1}^{n}\left(\frac{d_{c i}-d_{m i}}{d_{m i}}\right)^{2}} x 100
$$

dónde: $\mathrm{d}_{\mathrm{ci}}$ : deflexión calculada de un sensor $\mathrm{i}, \mathrm{d}_{\mathrm{mi}}$ : deflexión medida de un sensor $\mathrm{i}, \mathrm{n}_{\mathrm{d}}$ : número de sensores utilizado en el análisis.

- Tolerancia del módulo:

$$
E(\%)=\frac{E_{(k+1) i}-E_{(\mathrm{k}) \mathrm{i}}}{E_{(k) i}} \times 100
$$

donde: $\mathrm{E}_{(\mathrm{k}+1) \mathrm{i}}$ : módulo siguiente de una capa determinada $\mathrm{i}, \mathrm{E}_{(\mathrm{k}) \mathrm{i}}=$ módulo anterior de una capa determinada $i$.

- El máximo número de iteraciones con las que trabajará el programa, se recomiendan 10 iteraciones.

Modelización de la estructura del pavimento con elementos finitos

Las técnicas más avanzadas para el análisis y diseño racional de pavimentos consideran el uso de elementos finitos como la herramienta de mayor flexibilidad y versatilidad para la representación de los materiales, las condiciones de compatibilidad y la representación de capas. Para la presente investigación se utiliza el programa ANSYS para el análisis de elementos finitos (Suarez Dominguez, 
2001). Los modelos fundamentan su análisis en curvas de esfuerzo deformación de la teoría elástica multicapas en términos de estado de tensiones y deformaciones inducidas por diferentes solicitaciones generadas por factores como el tránsito y condiciones atmosféricas (Huang, 2004).

Proceso de construcción del modelo

Para la modelación se considera un semi-espacio lineal, elástico, isótropo y homogéneo. Dentro de ANSYS se pueden añadir capas de espesor variable y diferente tipo de material. Para cada capa que compone la estructura del pavimento se definen módulos elásticos y coeficientes de Poisson.

La estructura del pavimento dentro de la modelación se representa sólo la mitad derecha utilizando una formulación axisimétrica bidimensional. Para la modelación del pavimento se emplea el elemento SOLID 272 (véase Fig. 3) adecuado para modelar estructuras sólidas con simetría axial, el cual se define por cuatro nodos en el plano principal donde cada nodo tiene tres grados de libertad.

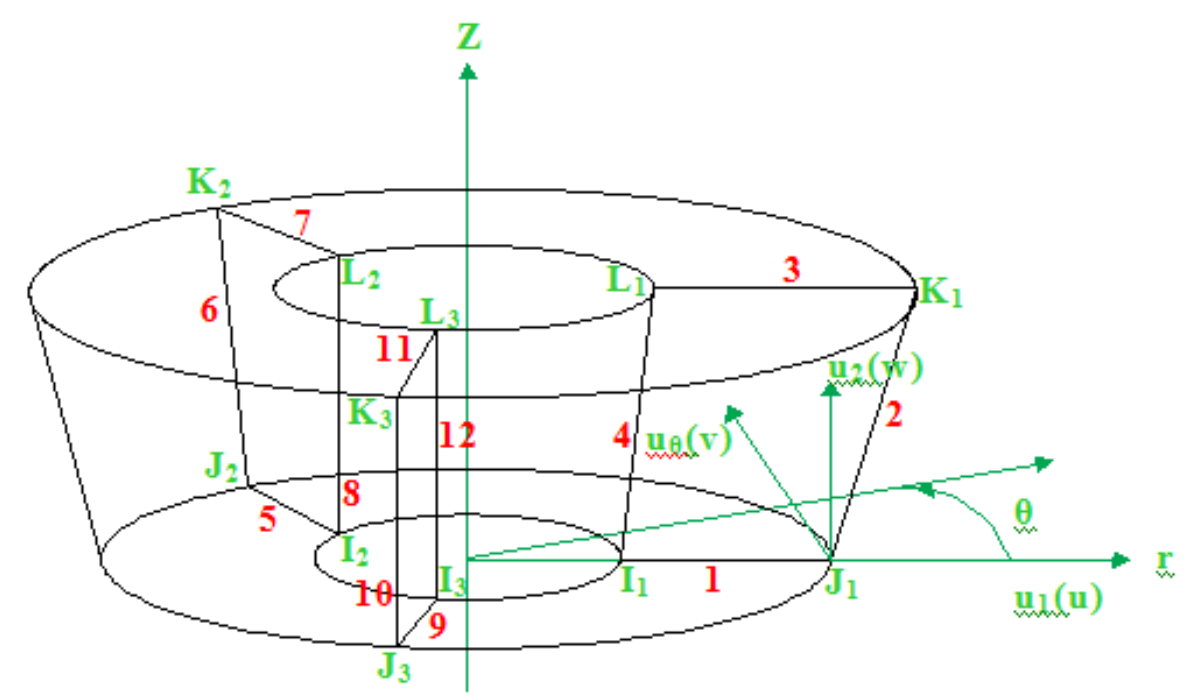

Figura 3. Elemento SOLID272.

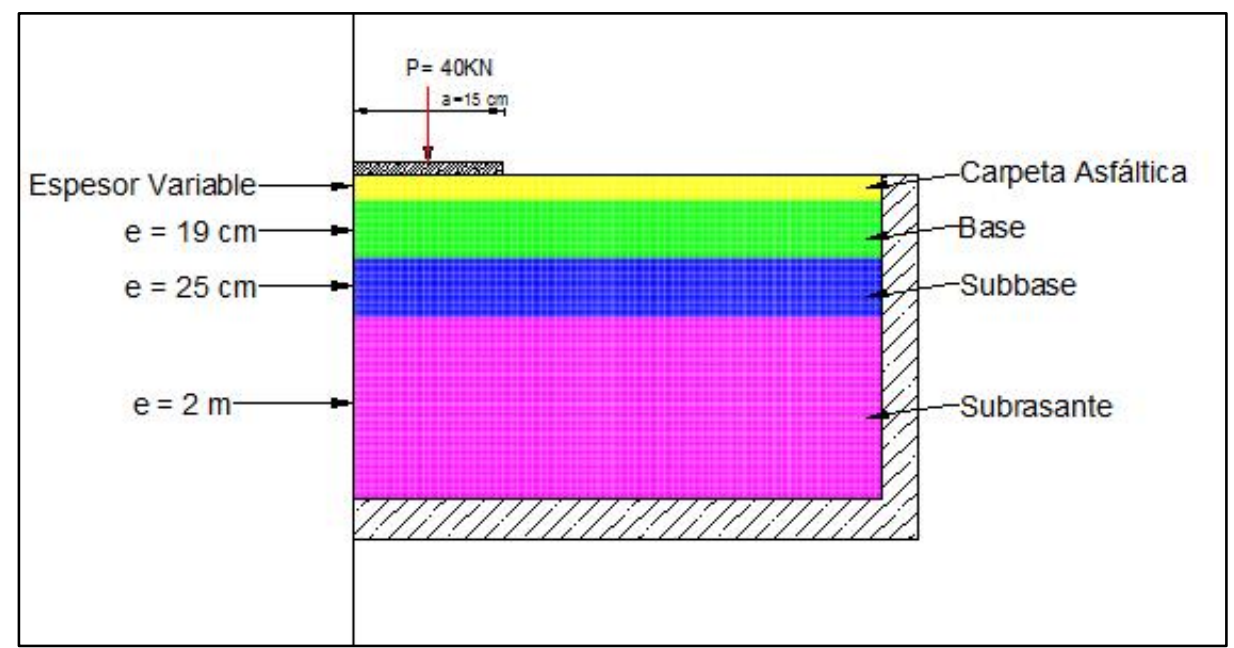

Figura 4. Modelización, geometría y mallado de los elementos.

Para modelar el ensayo del FWD se aplica una carga dinámica igual a $40 \mathrm{kN}$ sobre un área circular delimitada por el plato de carga el cual tiene un radio de $15 \mathrm{~cm}$, esto produce una presión de confinamiento igual a $566 \mathrm{kPa}$ que se aplica directamente sobre los nodos que se ubican en la parte superior de la estructura del pavimento, así se simula el plato de carga del FWD. La profundidad de 
confinamiento vertical de la estructura se establece en la medida que los desplazamientos de dicha base tiendan a cero, en teoría la subrasante se considera semi-infinita, sin embargo, en los modelos de elementos finitos se debe establecer líneas en donde los desplazamientos no sean importantes. Durante la investigación presente se consideró un espesor de 70 a $200 \mathrm{~cm}$. El confinamiento horizontal, debido a la simetría axial del modelo se establece a una distancia de $10 \mathrm{~m}$ medidos del eje del modelo.

Es necesario discretizar la estructura del pavimento mediante elementos cuadrados los que forman una malla. La Figura 4 muestra la modelización de la estructura.

\subsection{Metodología para la caracterización del pavimento}

Los ensayos de deflectometría se realizaron con el FWD PRI 2100 del Ministerio de Transporte y Obras Públicas (MTOP), cada $100 \mathrm{~m}$ alternando el carril, dando un total de 262 puntos de muestreo en todo el tramo de estudio. En cada punto de muestreo se ejecutaron 3 golpes sobre la vía; en donde el primero y el segundo se los realizo para calibrar el equipo, por tal motivo con el último golpe se obtuvo un total de 10 deflexiones, uno por cada geófono ubicado en la regleta. La información recopilada por el equipo fue analizada mediante los modelos matemáticos planteados en la Sección 2.3, caracterizando de este modo el sistema pavimento-subrasante.

Para la adecuada comparación entre las metodologías es necesario seccionar la vía en tramos cuyos parámetros geomecánicos sean uniformes; para lo cual se hace uso del método de las diferencias acumuladas propuesto por AASHTO 93. Esta herramienta visual permite seccionar el proyecto vial en base a la respuesta del pavimento que para el caso se usó la deflexión máxima de cada punto de muestreo (véase Fig. 5). Obteniéndose de tal modo tres secciones:

- Sección: 0+000 - 5+400, Esta sección cuenta con 57 puntos de ensayo, un valor de deflexión promedio igual a $230.98 \mu \mathrm{m}$

- Sección 2: 5+400 - 8+000, Esta sección cuenta con 24 puntos de ensayo, un valor de deflexión promedio igual a $334.56 \mu \mathrm{m}$

- Sección: 8+000 - 26+120, Esta sección cuenta con 181 puntos de ensayo, un valor de deflexión promedio igual a $267.28 \mu \mathrm{m}$

Como se puede observar en la Figura 5, la línea roja hace referencia al promedio de los valores en cada sección homogénea de análisis; en cambio la línea verde se refiere a la deflexión característica $\left(\mathrm{x}_{\mathrm{c}}\right)$; la que según AASHTO 93 equivaldría a un nivel de confianza del 95\%, es decir 1.65 veces la desviación estándar más el promedio en cada sección.

Mediante el seccionamiento de la vía se calcula el módulo resiliente de la subrasante y la capacidad estructural con cada uno de los modelos matemáticos. El módulo es analizado mediante la desviación estándar entre los 4 modelos, como una herramienta de selección de un conjunto de datos (deflexiones ubicadas a $0,20,30,40,60,90,120,150,180$ y $210 \mathrm{~cm}$, horizontalmente con respecto al eje de carga) que puedan ser ingresados a EVERCALC ya que el uso del mismo se limita a analizar cada punto de ensayo, lo que resulta poco práctico para el análisis, En la Figura 6 se expone el gráfico de las abscisas y el módulo resiliente de la subrasante determinado a través de EVERCALC, en cada una de las secciones establecidas.

Posteriormente, los módulos obtenidos en EVERCALC son ingresados en la modelización de elementos finitos que propone ANSYS, para lo cual se procede a trabajar con tres puntos representativos para la comparación de los resultados obtenidos de ANSYS y los valores reales del FWD (3 puntos que presenten valores de deflexión central aproximados a la media establecida por cada sección homogénea), dicha comparación se realiza con las deflexiones medidas en campo por el FWD y las calculadas mediante ANSYS (ver Tabla 4). 


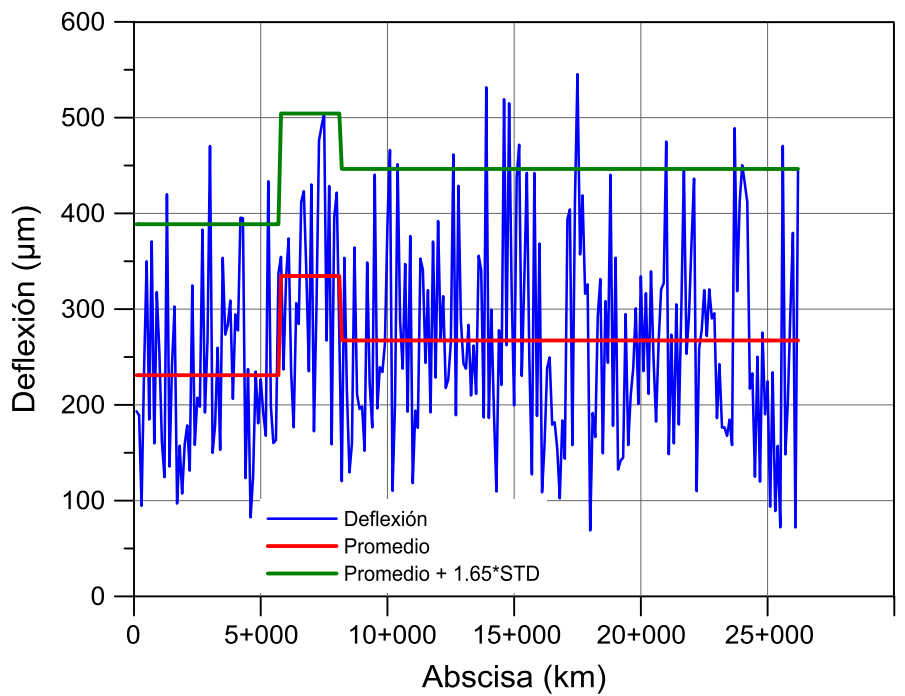

Figura 5. Secciones homogéneas de la vía Chicti - Sevilla de Oro.

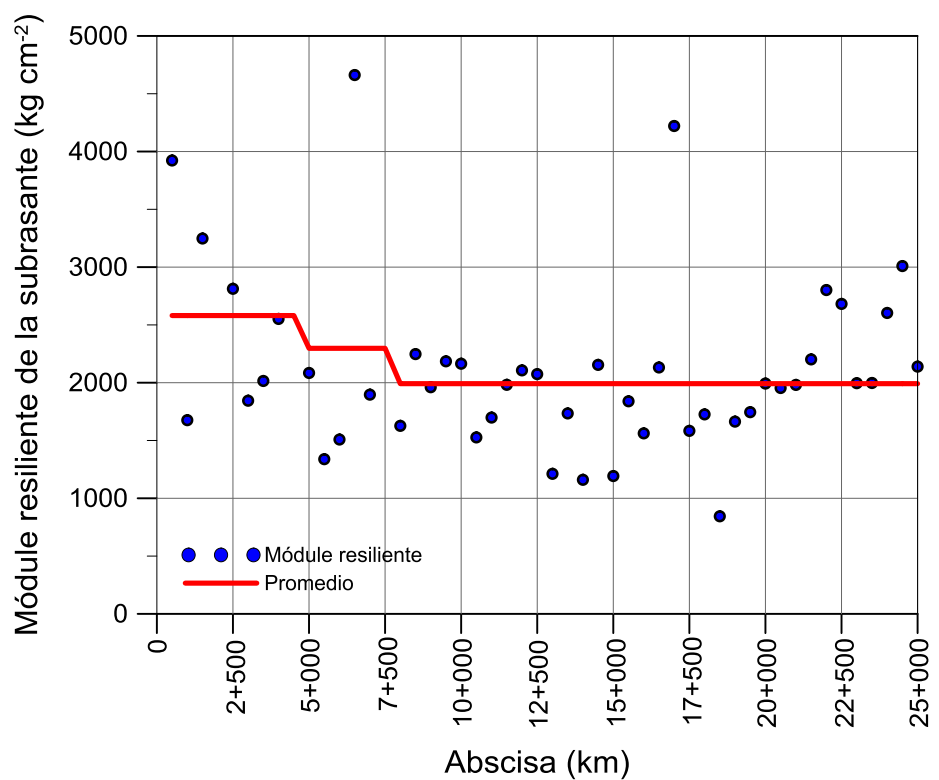

Figura 6. Módulo resiliente de la subrasante a través de EVERCALC.

Tabla 4. Deflexiones medidas por el FWD y calculadas por ANSYS.

\begin{tabular}{ccccccc}
\hline \multirow{2}{*}{$\begin{array}{c}\text { Distancia } \\
(\mathrm{cm})\end{array}$} & \multicolumn{9}{c}{ Sección 1 } & \multicolumn{2}{c}{ Seflexiones $(\mu \mathrm{m})$} \\
\cline { 2 - 7 } & \multicolumn{2}{c}{ Abscisa 1+500 } & \multicolumn{2}{c}{ Abscisa 6+300 } & \multicolumn{2}{c}{ Sección 3 } \\
& FWD & ANSYS & FWD & ANSYS & FWD & ANSYS \\
\hline 0 & 335 & 314 & 452 & 405 & 303 & 283 \\
20 & 276 & 250 & 358 & 310 & 246 & 215 \\
30 & 240 & 210 & 294 & 251 & 210 & 177 \\
40 & 206 & 175 & 239 & 203 & 179 & 147 \\
60 & 154 & 121 & 172 & 134 & 140 & 103 \\
90 & 97 & 68 & 108 & 72 & 93 & 61 \\
120 & 70 & 37 & 81 & 39 & 73 & 36 \\
150 & 49 & 19 & 56 & 22 & 54 & 22 \\
\hline
\end{tabular}


En la Figura 7 se observa que los cuencos de deflexiones calculados por ANSYS y medidos por el FWD, presentan una similitud en la forma con un desfase de aproximadamente $30 \mu \mathrm{m}$.
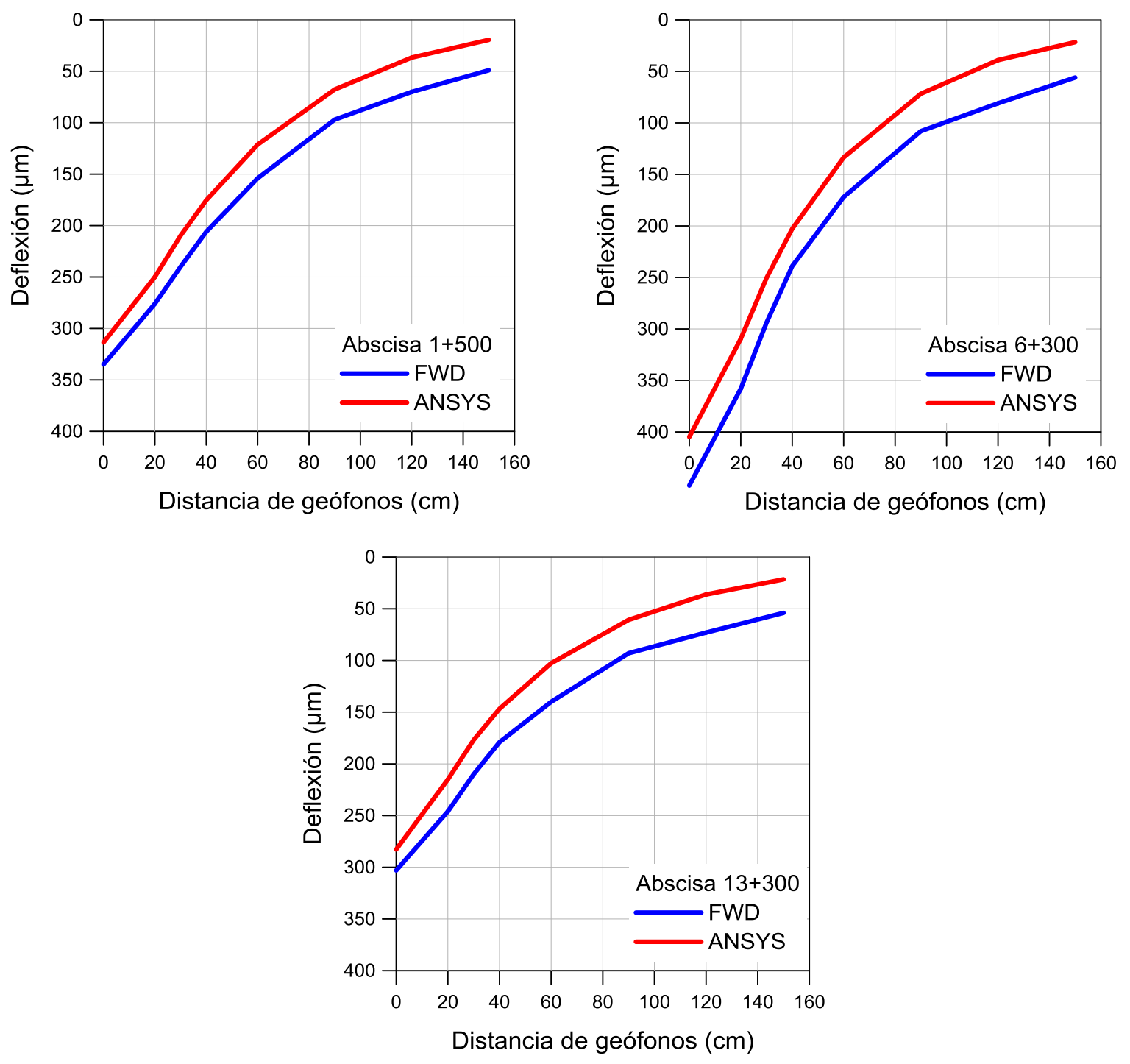

Figura 7. Deflexiones medidas por el FWD y calculadas por ANSYS.

\section{RESULTADOS Y DISCUSIONES}

Los valores de módulo resiliente obtenidos por las metodologías de AASHTO 93 y ROHDE son los más altos, los que no son comparables con las de YONAPAVE y LUKANEN (véase Fig. 8). Las metodologías que requieren del espesor de la estructura del pavimento $\left(\mathrm{H}_{\mathrm{T}}\right)$ generan resultados de módulo del orden de $60,000 \mathrm{~kg} \mathrm{~cm}^{-2}$, y además las ecuaciones para el módulo resiliente de la subrasante están en función de la deflexión ubicada a $180 \mathrm{~cm}$. Por este motivo, la similitud determinada entre AASHTO 93 y ROHDE establece un alto grado de correlación. Si bien las deflexiones ubicadas lo suficientemente lejos del punto central se relacionan con el cálculo del módulo resiliente, esto no quiere decir que las ubicadas a $180 \mathrm{~cm}$ puedan caracterizar de mejor forma al pavimento a través del módulo resiliente de subrasante. 


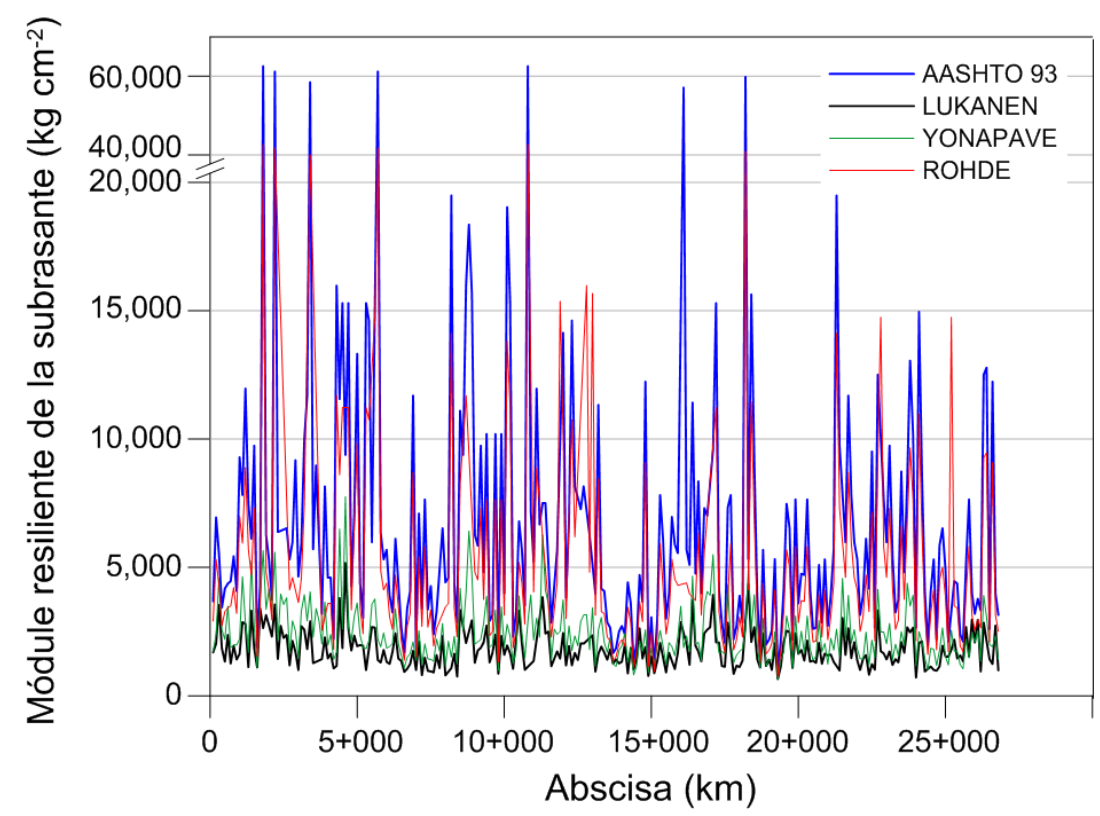

Figura 8. Módulo resiliente de subrasante entre los 4 modelos matemáticos.

Por otro lado, para las metodologías que plantean un análisis a través de las características del cuenco de deflexión (longitud característica y área del cuenco), los valores bordean los 7,000 a 8,000 $\mathrm{kg} \mathrm{cm}^{-2}$. La forma del cuenco de deflexiones resulta ser importante ya que pueden existir puntos de evaluación con deflexiones centrales aparentemente iguales, pero que al comparar deflexiones ubicadas a cierta distancia radial se pueden presentar variaciones, lo que implica una diferencia en la longitud característica. La metodología YONAPAVE plantea el análisis de la forma del cuenco de deflexiones a través del área, en donde considera varias deflexiones ubicadas a 0, 30, 60 y 90 cm; con el fin de caracterizar la estructura del pavimento.

Para el caso de la capacidad estructural (ver Fig. 9) se determina que los 4 modelos matemáticos presentan correlaciones del orden de $\mathrm{R}^{2}=0.80$. Sin embargo, mediante el análisis de pendientes de las rectas de ajuste se establece que existe una buena relación entre AASHTO 93 y LUKANEN (pendiente igual a 0.7957); pero por otro lado también se tiene la de YONAPAVE y ROHDE (pendiente igual a 0.8385 ).

Mediante la utilización del programa EVERCALC se ha realizado un análisis más detallado con el fin de caracterizar la estructura del pavimento a través del módulo resiliente de la subrasante. Mientras que mediante las 3 secciones homogéneas se establece tanto el módulo resiliente de la subrasante como el número estructural efectivo para cada una de las metodologías aplicando el mismo concepto de las diferencias acumuladas, para encontrar valores representativos en cada una de las secciones. Los resultados de este análisis se presentan en la Tabla 5. Los datos obtenidos por EVERCALC también son seccionados para llevarlos a valores representativos en cada uno de los tramos obtenidos por el concepto de deflexión máxima, esto con la finalidad de plantear el modelo matemático que mejor se ajuste los módulos resilientes de subrasante calculados por el programa.

El módulo resiliente de subrasante obtenido a través de las cuatro metodologías planteadas es comparado con el obtenido por el programa EVERCALC en cada una de las secciones que componen la longitud de la vía; con esto se establece que el modulo encontrado por AASHTO 93 es mayor. Aunque la metodología de ROHDE tiene resultados de módulo más bajos que los de AASHTO 93, el valor del módulo representativo se localiza comparando las metodologías de LUKANEN y YONAPAVE con el programa EVERCALC. Por ejemplo para el caso de la sección 1 el módulo calculado por YONAPAVE es de $2722.44 \mathrm{~kg} \mathrm{~cm}^{-2}$, y por EVERCALC es de $2581.16 \mathrm{~kg} \mathrm{~cm}^{-2}$. Por este motivo, en las tres secciones se puede observar que una de las metodologías que más se relaciona con el análisis de EVERCALC es la de YONAPAVE; por esto la evaluación estructural de la vía se hace 
aplicando el módulo resiliente de subrasante obtenido por el modelo matemático propuesto por YONAPAVE.
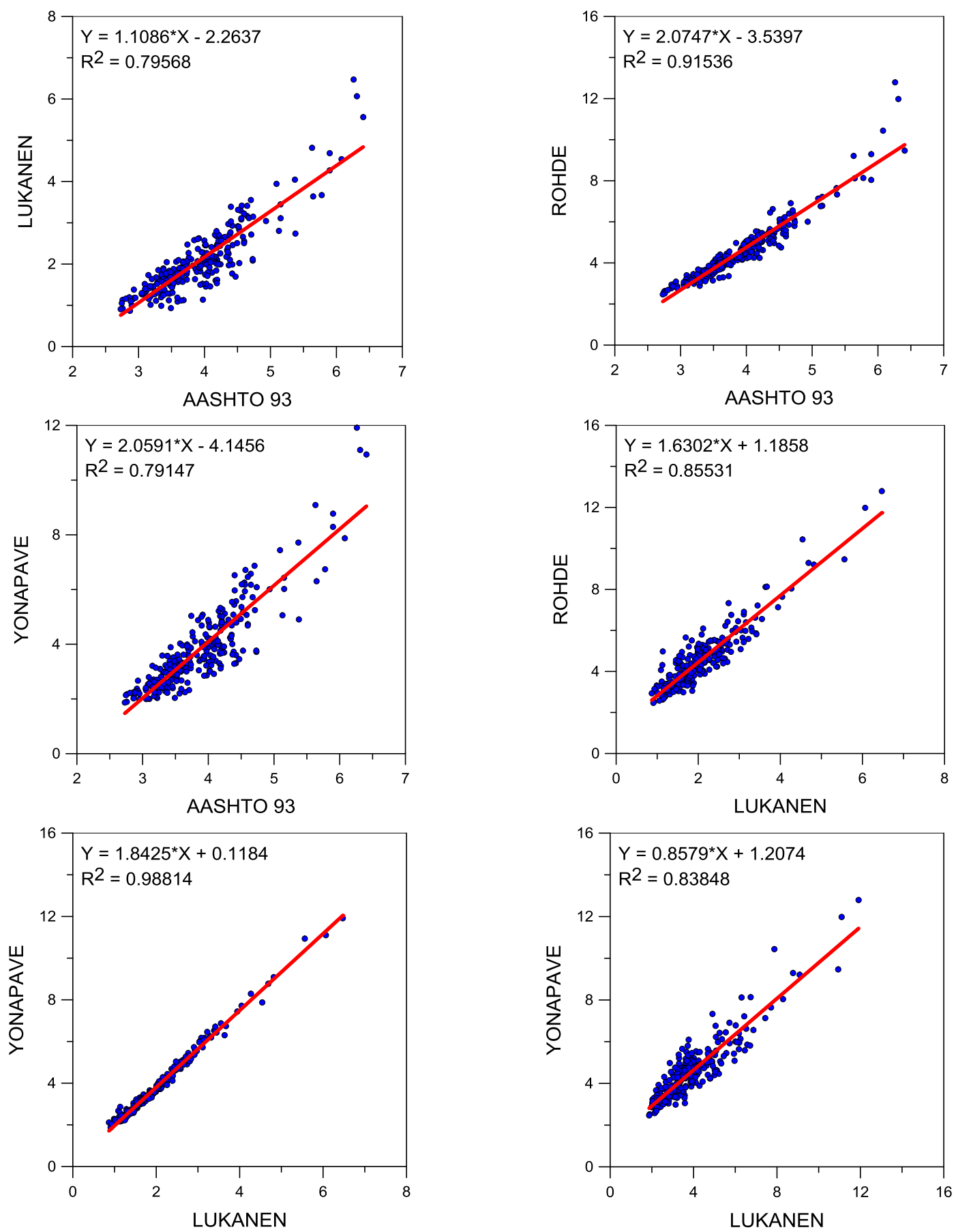

Figura 9. Correlación de $\mathrm{SN}_{\mathrm{ef}}$ entre los modelos matemáticos.

La comparación del número estructural calculado a través de cada modelo matemático se lo realiza con la aplicación del concepto de deflexión máxima característica $x_{c}=\bar{x}+1.65 \mathrm{~s}$ (donde $\bar{x}$ : promedio de la deflexión máxima en la sección analizada y s: desviación estándar en el mismo tramo) que permite calcular un número estructural efectivo que trabaja con un $95 \%$ de confiabilidad de los datos de deflexión máxima, método propuesto por AASHTO 93. En la Tabla 6 se presenta el cálculo 
del número estructural efectivo para un $95 \%$ de confiabilidad de la deflexión máxima corregida tanto por carga como por temperatura.

Tabla 5. Resultados del módulo resiliente y el número estructural a partir de análisis inverso.

\begin{tabular}{lcccccc}
\hline \multirow{2}{*}{$\begin{array}{c}\text { Metodologías } \\
\text { análisis inverso }\end{array}$} & \multicolumn{2}{c}{$\begin{array}{c}\text { Sección 1 } \\
0+000-5+400\end{array}$} & \multicolumn{2}{c}{$\begin{array}{c}\text { Sección 2 } \\
5+400-8+000\end{array}$} & \multicolumn{2}{c}{$\begin{array}{c}\text { Sección 3 } \\
8+000-26+120\end{array}$} \\
\cline { 2 - 7 } & $\begin{array}{c}\mathrm{M}_{\mathrm{rr}} \\
\left(\mathrm{kg} \mathrm{cm}^{-2}\right)\end{array}$ & $\mathrm{SN}_{\mathrm{ef}}$ & $\begin{array}{c}\mathrm{M}_{\mathrm{rr}} \\
\left(\mathrm{kg} \mathrm{cm}^{-2}\right)\end{array}$ & $\mathrm{SN}_{\mathrm{ef}}$ & $\begin{array}{c}\mathrm{M}_{\mathrm{rr}} \\
\left(\mathrm{kg} \mathrm{cm}^{-2}\right)\end{array}$ & $\mathrm{SN}_{\mathrm{ef}}$ \\
\hline AASHTO 93 & 8500.00 & 3.92 & 4693.97 & 3.48 & 7326.00 & 3.80 \\
LUKANEN & 1978.52 & 1.96 & 1300.00 & 1.79 & 1710.00 & 2.00 \\
YONAPAVE & 2722.44 & 3.65 & 2013.21 & 3.31 & 2254.45 & 3.66 \\
ROHDE & 6376.49 & 4.57 & 5169.91 & 3.83 & 5294.21 & 4.20 \\
EVERCALC & 2581.16 & & 2297.91 & & 1991.33 & \\
\hline
\end{tabular}

Tabla 6. Análisis del número estructural efectivo a través de la deflexión característica.

\begin{tabular}{lccc}
\hline & Sección 1 & Sección 2 & Sección 3 \\
\hline $\mathrm{h}_{1}(\mathrm{~cm})$ & 7.95 & 7.31 & 8.54 \\
$\mathrm{x}_{\mathrm{c}}(\mu \mathrm{m})$ & 388.65 & 504.30 & 446.39 \\
$\mathrm{M}_{\mathrm{rr}}\left(\mathrm{kg} \mathrm{cm}^{-2}\right)$ & 8500.00 & 4693.97 & 7326.00 \\
$\mathrm{E}_{\mathrm{p}}\left(\mathrm{kg} \mathrm{cm}^{-2}\right)$ & 2793.19 & 2216.35 & 2500.23 \\
$\mathrm{SN}_{\mathrm{ef}}$ & 3.14 & 3.17 & 3.37 \\
\hline
\end{tabular}

donde: $\mathrm{SN}_{\mathrm{ef}}=$ número estructural efectivo, $\mathrm{Xc}=$ deflexión característica, $\mathrm{M}_{\mathrm{rr}}=$ módulo resiliente de la subrasante obtenido por AASHTO 93, Ep = módulo equivalente del pavimento, y $\mathrm{h}_{1}=$ espesor de la carpeta asfáltica.

Al compararse la capacidad estructural efectiva entre cada uno de los modelos con el valor del número estructural efectivo, se observa que la metodología que mejor se ajusta es la de YONAPAVE. Por ejemplo para el caso del Tramo 2 se establece una diferencia de 3.31-3.17=0.14, lo que para las otras metodologías se obtienen diferencias mayores.

\section{EVALUACIÓN DEL ESTADO ACTUAL DE LA VÍA}

Las cargas producidas por el tránsito actual de la vía sirven para evaluar la condición del pavimento. Por este motivo, el tránsito actual de la vía se obtuvo de la base de datos del Ministerio de Transporte y Obras Públicas (MTOP), dentro de este informe se encuentra el TPDA (Tráfico Promedio Diario Anual) para el año 2013, el cual corresponde a 10,160. La vía Chicti-Sevilla de Oro cuenta con dos tipos de vehículos representativos de acuerdo a la disposición de sus ejes 2DB (Camión de 2 ejes, con 2 ruedas delanteras y 4 traseras) y 3-A (Camión de 3 ejes, con un eje delantero y 2 ejes posteriores). El factor de equivalencia de carga (FE) para los vehículos 2DB es de 3.8 que tiene una composición de $13.42 \%$ del total de vehículos comerciales y para los vehículos 3-A el valor de FE es igual a 3.5 con una composición de $2.08 \%$. Con los valores mencionados, se obtiene el factor camión (FC) mediante una media ponderada de los factores de equivalencia, dando como resultado $3.76 \%$.

Se procede a transformar al tránsito a un número de ejes equivalentes $\left(\mathrm{W}_{18}\right)$ de una carga de 18 Kips que producirán el mismo daño que toda la composición del tránsito. Para ello se tendrá en cuenta diferentes aspectos tales como: la vía en estudio cuenta con 2 carriles por lo que el factor de distribución direccional es del $50 \%$ y un factor de distribución por carril del 100\%, la capa de rodadura es de concreto asfáltico, nivel de confiabilidad de $95 \%$ lo que da un valor de 1.65 para la desviación estándar, la pérdida del nivel de servicio durante el periodo de diseño, considerando un 
nivel de serviciabilidad inicial de 4.2 y un final de 2; y un porcentaje de vehículos comerciales correspondiente a $15.50 \%$. La tasa de crecimiento para camiones determinada en la provincia del Azuay en del período $2010-2015$ es del 3.37\%. Con la Ec. 22 se obtiene el número de ejes equivalentes para la vía en estudio, el cual tiene una magnitud de 1,080,107 de ejes equivalentes. Cabe mencionar que el número de ejes equivalentes obtenido es el esperado para el año 2014, es decir se proyectó al tráfico para un año.

$$
W_{18}=T P D A^{*} F d * F c * \mathrm{~F}_{\text {proy }} * \mathrm{FC} * \% \mathrm{Vc} * 365
$$

donde: $\mathrm{W}_{18}$ es el número estimado de ejes simples equivalentes de $18 \mathrm{Kips} ; \mathrm{F}_{\mathrm{d}}$ es el factor de distribución direccional igual a 50\%; $\mathrm{F}_{\mathrm{c}}$ es el factor de distribución por carril del $100 \%$; $\mathrm{F}_{\text {proy }}$ es el factor de proyección propuesto por AASHTO 93 (tiempo de proyección es 1 año), y \% Vc es el porcentaje de vehículos comerciales equivalente a $15.50 \%$.

Mediante las 3 secciones establecidas y los módulos resilientes obtenidos mediante el método de YONAPAVE (véase Tabla 7), se procede a calcular el valor del número estructural requerido por el tráfico $\left(\mathrm{SN}_{\mathrm{f}}\right)$, mediante la Ec. 23:

$$
\log W_{18}=Z r * S o+9.36 \log \left(S N_{f}+1\right)-0.2+\frac{\log \left(\frac{\Delta P S I}{4.2-1.5}\right)}{0.4+\left(\frac{1094}{\left(S N_{f}+1\right)^{5.19}}\right)}+2.32 \log \left(M_{r}\right)-8.07
$$

donde: $\mathrm{Z}_{\mathrm{r}}$ : Desviación estándar normal; So:Error estándar combinado de la predicción del tránsito y de la predicción del comportamiento; $\triangle \mathrm{PSI}$ :Diferencia entre el índice de servicio inicial $(\mathrm{Po}=4.2)$ y el final ( $\mathrm{Pt}=2.0) ; \mathrm{M}_{\mathrm{r}}$ :Modulo Resiliente de la subrasante y $\mathrm{SN}_{\mathrm{f}}$ :Número Estructural requerido por el Tráfico Actual.

Tabla 7. Determinación de la capacidad estructural $\left(\mathrm{SN}_{\mathrm{f}}\right), * \mathrm{M}_{\mathrm{rr}}=$ Módulo Resiliente obtenido mediante YONAPAVE.

\begin{tabular}{cccc}
\hline $\mathrm{W} 18$ & $1,080,107$ & So & 0.45 \\
$\mathrm{Zr}$ & 1.65 & $\Delta$ PSI & 2.20 \\
\hline & Sección 1 & Sección 2 & Sección 3 \\
\hline$* \mathrm{M}_{\mathrm{rr}}\left(\mathrm{kg} \mathrm{cm}^{-2}\right)$ & 2722.44 & 2013.21 & 2254.45 \\
$\mathrm{M}_{\mathrm{r}}\left(\mathrm{kg} \mathrm{cm}^{-2}\right)$ & 898.41 & 664.36 & 743.97 \\
$\mathrm{SN}_{\mathrm{f}}(\mathrm{Número} \mathrm{Estructural} \mathrm{Futuro})_{\mathrm{SN}_{\mathrm{ef}}(\text { YONAPAVE) }}$ & 2.02 & 2.26 & 2.17 \\
\hline
\end{tabular}

El número estructural es un parámetro importante en el diseño de espesores para pavimentos flexibles, por este motivo es imprescindible diferenciar en función de que variables se encuentra. Por un lado, el número estructural se encuentra $\left(\mathrm{SN}_{\mathrm{f}}\right)$ en función de variables de serviciabilidad, módulo resiliente de subrasante pero principalmente del tráfico que ha de circular por la vía para un periodo de diseño determinado, y al cual se lo interpreta con el número de ejes equivalentes. Por otra parte, se distingue la capacidad estructural $\left(\mathrm{SN}_{2}\right)$ en función de coeficientes de capa, coeficientes de drenaje y espesores que componen la estructura del pavimento.

Para el diseño, se debe tener presente que el $\mathrm{SN}_{2}$ debe ser mayor o igual que el $\mathrm{SN}_{\mathrm{f}}$, esto con el fin de asegurar que la vía presente condiciones óptimas de serviciabilidad durante toda su vida útil para la cual fue proyectada.

La relación de lo expuesto anteriormente con la evaluación de pavimentos mediante deflectometría de impacto se da en el hecho de que la capacidad estructural efectiva que es calculada mediante modelos matemáticos basados en análisis inverso $\left(\mathrm{SN}_{\mathrm{ef}}\right)$ sería para este caso el $\mathrm{SN}_{2}$. Desde el punto de vista económico, si la variable $\mathrm{SN}_{\mathrm{f}}$ es mayor que $\mathrm{SN}_{\mathrm{ef}}$ se establece que sería necesario una rehabilitación, y es por esto que la diferencia de las mismas se encuentra en función de los espesor de 
sobrecarpetas que debería ser construidas para mejorar el nivel de servicio de una vía y por ende de los costos que implicaría ejecutar dicha rehabilitación.

Para el caso de la investigación realizada, a través del número estructural efectivo obtenido por la deflexión máxima característica y el número estructural obtenido mediante la relación del tráfico presente en la vía se establece en la Tabla 8 , en la que se puede observar que el desempeño estructural de la vía es adecuado.

\section{CONCLUSIONES Y RECOMENDACIONES}

La aplicación del FWD para caracterizar el comportamiento de una estructura de pavimento, la cual se encuentra sometida a cargas de tránsito es un procedimiento no destructivo sencillo y práctico.

El análisis de los datos provenientes del FWD (deflexiones) mediante el módulo resiliente obtenido entre las 4 metodologías AASHTO 93, ROHDE, LUKANEN y YONAPAVE y con el software EVERCALC; permite concluir que existe una adecuada tendencia de los resultados por el programa con los modelos de LUKANEN Y YONAPAVE, los cuales no requieren del espesor de toda la estructura, por esto se establece que una adecuada caracterización del pavimento lo proporcionan metodologías que estudian las características del cuenco de deflexiones.

Con el fin de contrastar los resultados obtenidos por EVERCALC se modelaron los resultados en ANSYS mediante el análisis de tres puntos representativos correspondientes a las abscisas $1+500$, $6+300$ y 13+300, uno por cada sección homogénea de la vía, con el ingreso de módulos elásticos de cada capa establecidos por el programa EVERCALC. El modelamiento a través de elementos finitos del sistema pavimento - subrasante permite establecer que el cuenco de deflexiones obtenido por ANSYS es similar al cuenco de deflexiones del FWD, en la que el ajuste de las curvas de deflexión marca una variación que puede ser justificada en el hecho de que programas como EVERCALC son herramientas computacionales desarrolladas para un área específica de la Ingeniería de Pavimentos como lo es la evaluación estructural de pavimentos mediante deflectometría de impacto.

A través de los resultados y estadísticas obtenidos se concluye que el modelo matemático basado en análisis inverso más adecuado para la caracterización del sistema pavimento-subrasante en la vía en estudio es YONAPAVE. Una ventaja de este método es que no necesita de los espesores de capas del pavimento, y por ende no se necesita realizar procesos destructivos como las calicatas para obtener el espesor total de la estructura del pavimento, ya que este modelo trabaja con el concepto del área del cuenco de deflexiones generado por la aplicación de una carga dinámica proveniente del FWD.

Los resultados obtenidos de la evaluación estructural de la vía dan una idea general de la condición actual. Con lo que se puede concluir que actualmente la vía presenta condiciones aceptables ya que a través de la deflectometría de impacto aplicando técnicas de análisis inverso como la de YONAPAVE se ha comparado la capacidad estructural efectiva del pavimento existente con la capacidad estructural que depende del tráfico presente en la vía.

En la actualidad, el estudio de modelos matemáticos basados en análisis inverso que permiten caracterizar un adecuado comportamiento de la estructura del pavimento que se encuentra sometida principalmente a las cargas del tránsito debe ser indagado con mayor detalle, ya que si bien el uso de la metodología AASHTO 93 es ampliamente utilizado en Ecuador, esto no quiere decir que sea la alternativa más viable.

\section{BIBLIOGRAFÍA}

AASHTO, 1993. AASHTO guide for design of pavement structures. American Association of State Highway and Transportation Officials, Washington, D.C. Disponible en https://habib00ugm.files.wordpress.com/2010/05/aashto1993.pdf, 624 pp. 
ASTM Standards, 2003. Standard guide for general pavement deflection measurements. ASTM D4695-03, Standard Guide for General Pavement Deflection Measurements, ASTM International, West Conshohocken, PA, 2003, www.astm.org.

ASTM International, 2003. Standard test method for deflections with a falling-weight-type impulse load device. ASTM Standards: number D 4694-96. Disponible en http://library.sut.ac.th:8080/ astm/cd04032005/PDF/D4694.pdf, 3 pp.

Asphalt Institute, 2000. Manual Series-17: Asphalt overlays for highway and street rehabilitation $\left(9^{\text {th }}\right.$ ed.). Disponible en http://www.asphaltinstitute.org/wp-content/uploads/public/engineering/ SW_1_Users_Guide_ver_103.pdf.

Corros, M., E. Urbaéz, G. Corredor, 2009. Manual de evaluación de pavimentos. Empresa MAYER 97 Ingenieros Asociados, C.A. Disponible en https://sjnavarro.files.wordpress.com/2008/08/ manual-de-evaluacion1.pdf, 249 pp.

del Aguila Rodríguez, P., 2007. Determinación del módulo resiliente del suelo de fundación mediante método mecanistico-empiricista: Método Hogg Simplificado. Ponencia presentada al XIV Congreso Ibero Latinoamericano del Asfalto, La Habana, Cuba. Disponible en http://www.camineros.com/docs/cam057.pdf, 17 pp.

Guzmán Suárez, E.A., 2009. Evaluación estructural de pavimentos flexibles a partir de información tomada con el deflectometro de impacto. XVII Simposio Colombiano sobre Ingeniería de Pavimentos, Universidad EAFIT, Colombia

Higuera Sandoval,C.H., 2010. Caracterización de la resistencia de la subrasante con la información del deflectómetro de impacto. Dialnet, 19(28), 73-92.

Hoffman, M.S., 2003. A direct method for evaluating the structural needs of flexible pavements based of FWD deflections. YONA, Engineering Consulting \& Management Ltd., Haifa, Israel. Disponible en http://www.academia.edu/9968038/A_Direct_Method_for_Evaluating_ the_Structural_Needs_of_Flexible_Pavements_Based_on_FWD_Deflections, 13 pp.

Huang, Y.H., 2004. Pavement analysis and design ( $2^{\text {nd }}$ ed.). Prentice-Hall, Englewood Cliffs, NJ.

Liu, W., T. Scullion, 2001. Modulus 6.0 for Windows: User's Manual. Texas Transportation Institute, Austin, Texas. Disponible en http://d2dt15nnlpfr0r.cloudfront.net/tti.tamu.edu/documents/18692.pdf, $52 \mathrm{pp}$.

Lukanen, E.O., C. Han, 1992. Pavement performance prediction models. Minnesota: Department of Transportation. Publisher: Braun Intertec Pavement, 121 pp.

Montejo Fonseca, A., 2002. Ingenieria de pavimentos para carreteras ( $2^{\text {nd }}$ ed.). Bogotá, D.C: Agora Editores.

Murillo Feo, C.A., L.E. Bejarano Urrego, 2013. Correlation between deflections measurements on flexible pavements obtained under static and dynamic load techniques. Proceedings of the $18^{\text {th }}$ International Conference on Soil Mechanics and Geotechnical Engineering, Paris, pp. 393-398. Disponible en http://www.cfms-sols.org/sites/all/lic/pages/download_pdf.php?file=393-398.pdf.

Roa Garzón, M.A., D.A. Garzón Alvarado, 2012. Introducción al modelamiento por elementos finitos con ANSYS. Bogotá: Departamento de Ingeniería Mecánica y Mecatrónica, Facultad de Ingeniería, Universidad Nacional de Colombia.

Rohde, G.T., 1994. Determining pavement structural number from FWD testing. In: Transportation Research Record 1448. TRB, National Research Council, Washington, D.C., pp. 61-68.

Rodriguez Calderón, W., M.R. Pallarez Muñoz, 2009. Desarrollo de un modelo de elementos finitos para el diseño racional de pavimentos. Tecnura, 9(17), 26-37.

Suarez Dominguez, F.J., 2001. Manual de diseño de ANSYS. España.

WSDOT, 2005. Everseries User Guide: Pavement analysis computer software and case studies. Washington State Department of Transportation, Enviromental and Engineering Programs, Washington, D.C. Disponible en http://www.wsdot.wa.gov/NR/rdonlyres/0C02BB7B-C3454958-AA08-089E5E512B96/0/EverseriesUserGuidePart1.pdf, 122 pp. 